\title{
Stress Echocardiography in Valvular Heart Disease
}

\author{
Sveeta Badiani a, b *, Peter Waddingham ${ }^{a, b}$ *, Guy Lloyd a,b,c,d, Sanjeev \\ Bhattacharyya ${ }^{a, b, c, d}$
}

aEchocardiography Laboratory, Barts Heart Centre, St Bartholomew's Hospital, London, UK

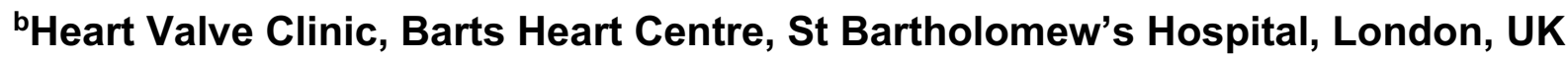
'William Harvey Research Institute, Queen Mary \& Westfield, University of London, UK

d Institute of Cardiovascular Sciences, UCL, London, UK

* joint first authors

Word count:

Address for correspondence:

Dr Sanjeev Bhattacharyya MD MRCP FESC

Heart Valve Clinic \& Echocardiography Laboratory,

Barts Heart Centre, St Bartholomew's Hospital

London, United Kingdom

Telephone: +44 (0)203416500

E-mail: Sanjeev.Bhattacharyya@bartshealth.nhs.uk 
There is growing recognition that assessment of heart valve disease in a resting state provides an incomplete evaluation of valve haemodynamics as the severity of valve obstruction and regurgitation is dynamic. Changes in flow and alterations in afterload, pre-load and heart rate during both pharmacological stress and exercise can change the valve haemodynamics. In low flow states, incrementing stroke volume may reveal the true severity of aortic stenosis. Alterations in opening and closing forces on the mitral valve with exercise alters the degree of mitral regurgitation/stenosis and may explain the cause of symptoms in patients with only mild regurgitation/stenosis at rest. This review examines the role of stress echocardiography in heart valve disease focussing on the pathophysiology of changes in valve haemodynamics during stress echocardiography and how these can be used to improve our quantification of valve disease, explain symptoms and aid decision making for valve intervention and follow-up.

\section{AORTIC STENOSIS}

Aortic stenosis is the most common left sided valve lesion [1] and affects more than $10 \%$ of individuals over the age of 75 [2]. Symptomatic severe aortic stenosis or lowgradient aortic stenosis with reduced ejection fraction and evidence of contractile reserve are Class I indications for aortic valve intervention [3,4]. Surgical aortic valve replacement is indicated in asymptomatic patients with severe aortic stenosis and left ventricular ejection fraction (LVEF) $<50 \%$ or if symptoms related to aortic stenosis occur on exercise testing $[3,4]$. However, there are many areas where controversy regarding the optimal management of patients is debated. These include the management of patients with asymptomatic, severe aortic stenosis and the diagnosis and management patients with severe aortic stenosis with an aortic valve area $<1 \mathrm{~cm}^{2}$ but a mean gradient $<40 \mathrm{mmHg}$. 
Figure 1 provides a diagnostic algorithm for the use of stress echocardiography in aortic stenosis.

\section{Asymptomatic SeVere Aortic Stenosis}

The timing of intervention in asymptomatic severe aortic stenosis is controversial. The clinical course is highly variable with a risk of sudden death (1-1.5\% per year) $[5,6,7]$. Regular surveillance with six monthly clinical and echocardiographic evaluation is currently recommended [3]. Early surgery requires consideration of both the risk of surgery and prosthetic valve related complications. Nearly $40 \%$ of patients with severe aortic stenosis do not report symptoms at the time of diagnosis but limitation is unmasked during exercise treadmill testing. Contrary to previous guidance exercise testing has been shown, comprehensively, to be safe in these patients and the results provide prognostic information $[8,9,10]$. Exercise parameters of value include exercise induced symptoms, a fall in blood pressure, ST depression and ventricular arrhythmia [8]. A more parametric approach including quantification of exercise workload may be useful; Masri et al [11] found, in 533 asymptomatic patients with severe aortic stenosis and preserved left ventricular ejection fraction undergoing exercise stress echocardiography with 6 year follow up, a lower percentage of age-sex-predicted metabolic equivalents (METS) and slower heart rate recovery were associated with longer-term mortality.

Exercise stress echocardiography allows not just the assessment of functional capacity and haemodynamic changes but also the measurement of transvalvular gradient under higher flow conditions, measurement of left ventricular contractile function and estimation of pulmonary artery systolic pressures. In asymptomatic severe aortic stenosis, a stiffer, less compliant valve (as evidenced by a significant exercise induced 
rise in mean aortic valve gradient of $18-20 \mathrm{mmHg}$ ) has been demonstrated to be a marker of increased likelihood of aortic valve related events during follow-up [12,13]. The increase in mean gradient during exercise may reflect either the presence of more severe aortic stenosis or a non-compliant rigid valve. Lancellotti et al [14] also identified exerciseinduced pulmonary hypertension occurring in $55 \%$ of their cohort of patients with asymptomatic severe aortic stenosis and was associated with a two fold increased risk of cardiac events. Although this data has been challenged by Goublaire et al [15]. Among 148 patients undergoing exercise stress echocardiography, 112 patients had a normal exercise test with no symptoms, fall in blood pressure and/or ST segment depression. Out of these, 38 patients (34\%) exhibited a mean gradient increase $>20 \mathrm{mmHg}$ and/or systolic pulmonary artery pressure (SPAP) $>60 \mathrm{mmHg}$ at peak exercise. During a mean follow up of $14 \pm 8$ months, neither a mean gradient increase $>20 \mathrm{mmHg}$ nor peak SPAP $>60 \mathrm{~mm} \mathrm{Hg}$ was predictive of occurrence of aortic stenosis-related events or aortic valve replacement.

An abnormal left ventricular response to exercise (manifest by a lack of increment or decrease in LVEF on exercise) is associated with increased likelihood of developing symptoms on exercise and lower survival free of cardiac events than those with an appropriate increase in LVEF on exercise [16]. However, left ventricular longitudinal strain is a more powerful parameter than LVEF to predict the occurrence of symptoms, exercise tolerance and cardiac events $[17,18]$. Huded et al [19], in a study of 504 asymptomatic patients with severe aortic stenosis, showed that age and sex predicted METS (hazard ratio 1.16), left ventricular global longitudinal strain (hazard ratio 1.12) and valvuloarterial impedance (Zva) (hazard ratio 1.25) offered incremental prognostic value. However, at 
present no clinically applicable cut-offs for contractile reserve using strain in aortic stenosis have been validated.

All of these studies have included a relative small number of patients and the conflicting results mean further research into the significance of valve gradient changes, contractile reserve and changes in pulmonary vascular behaviour is required. Despite the lack of conclusive data the objective assessment of the myocardial and valve behaviour under stress conditions, especially when combined with objective measures of exercise capacity is helpful in the assessment of patients where decision making is difficult.

\section{Test PROTOCOL}

Performing the test using a semi-supine bicycle is generally the preferred method of performing the test, as images can be acquired during exercise, rather than immediately after exercise [20]. Baseline images must include an assessment of all left ventricular myocardial segments, aortic valve continuous wave Doppler for measurement of mean gradient, pulse wave velocity from the left ventricular outflow tract and continuous wave doppler across the tricuspid valve for the estimation of PASP. A 12 lead electrocardiogram should be placed to look for ST segment changes and arrhythmia. Blood pressure measurements should be made at each stage. There is no unified and excepted protocol however a workload of 25 Watts increasing in 25 Watts increments at 2 minute intervals is usually recommended. The test is symptom limited and stopped if the patient develops dyspnoea, chest pain, dizziness or there are adverse haemodynamic changes (reduction in blood pressure, arrhythmia, ST changes). Images should be 
acquired at an intermediate heart rate of between 90 and 110 beats per minute as well as at peak exercise.

\section{LoW FLow, Low GRADIENT AORTIC STENOSIS}

Severe high-gradient aortic stenosis is conventionally defined as a calculated aortic valve area of $<1.0 \mathrm{~cm}^{2}$ (or $<0.65 \mathrm{~cm}^{2} / \mathrm{m}^{2}$ ) corroborated by a peak aortic jet velocity of $>4.0 \mathrm{~m} / \mathrm{s}$ and a mean transvalvular pressure gradient of $>40 \mathrm{mmHg}$ [21]. The classification of aortic stenosis severity is not always so straightforward, and the echocardiographic findings are discordant in one in three patients [22,23]. The most common being a valve area in the severe range $(<1 \mathrm{~cm} 2)$ with a low mean gradient $(<40 \mathrm{mmHg})$. Here, the first and most important distinction needs to be made between

those with low flow (conventionally considered $<35 \mathrm{mls} / \mathrm{m} 2$ ) and those with normal flow, Amongst the latter in whom measurement inaccuracy is most commonly a source of error [21] as well as problems with the assumptions of circularity in the often eccentric LVOT and the inherent discrepancy between valve area and gradient $[23,24]$.

Once satisfied that the situation is one of low-flow low-gradient (LF-LG), patients can be further sub classified into those with a reduced LVEF (defined as classical low flow) or preserved LVEF (termed paradoxical low flow). In the former ventricular systolic function is usually the principle cause of the low flow while the latter is more complex with a contribution from myocardial geometry (small left ventricular cavity size), ventricular long axis dysfunction and augmented afterload. The evidence for classical LFLG is very robust whereas the role of stress echo in the latter is much less clear.

\section{LoW-Flow, LoW-Gradient Aortic Stenosis With Reduced EJection Fraction}


When the LVEF is reduced it is important to distinguish between true-severe aortic stenosis with low flow (where severe aortic stenosis and high afterload are the main causes of the reduced flow) and so called pseudo-severe aortic stenosis (mild or moderate aortic stenosis with low flow predominantly due to left ventricular myocardial disease leading to incomplete aortic valve opening and a reduced valve area) $[25,26]$.

Dobutamine stress echocardiography (DSE) provides robust data to differentiate between these by increasing flow through the valve [25]. By augmenting transvalvular flow dobutamine infusion there should be an increase in stroke volume. An increase of $>20 \%$ suggests the presence of flow reserve. There are three principle outputs from Dobutamine stress echo. True-severe aortic stenosis where the typical findings will be an unchanged aortic valve area (which remains $<1 \mathrm{~cm}^{2}$ ) but a marked increase in mean gradient to $>40 \mathrm{mmHg}$; pseudo-severe (moderate) AS where there is an increase in valve area but relatively little increase in mean gradient [27]. In the third output flow reserve is not observed making differentiation between severe and pseudo-severe aortic stenosis difficult. One approach presented by the TOPAS study investigators is to calculate a projected effective orifice area that would have occurred at a standardised flow rate of $250 \mathrm{ml} / \mathrm{s}$. This has the advantage that, while stroke volume does have to increase to some extent, an increase in $20 \%$ is not required They have shown projected aortic valve area is more closely related to actual aortic stenosis severity and a better marker of prognosis and than traditional DSE parameters $[28,29]$.

The assessment of left ventricular flow reserve during low-dose DSE has clear prognostic implications in true severe aortic stenosis [30,31]. If a $20 \%$ increase in stroke volume is associated with the development of a mean aortic gradient of $40 \mathrm{mmHg}$ or a 
peak velocity of greater than $4 \mathrm{~m} / \mathrm{s}$, with an unchanged valve area, then the aortic stenosis is severe and intervention is warranted $[3,4]$. If no flow recruitment is observed then the outlook from surgery is very poor, with a high operative risk, and the recommendations for aortic valve replacement in these patients is weaker (llb, level of evidence C) [3,4].

\section{Low Flow Low Gradient Aortic Stenosis With Preserved EJection Fraction}

Paradoxical low-flow low-gradient aortic stenosis occurs in $25-35 \%$ of patients with low-gradient aortic stenosis $[32,33]$. It is defined as an aortic valve area $<1.0 \mathrm{~cm}^{2}$, indexed aortic valve area $<0.6 \mathrm{~cm}^{2} / \mathrm{m}^{2}$, mean gradient $<40 \mathrm{mmHg}$, LVEF $\geq 50 \%$, and presence of low flow (stroke volume index $<35 \mathrm{ml} / \mathrm{m}^{2}$ ). The low stroke volume is usually caused by a small left ventricular cavity related to concentric left ventricular hypertrophy compounded by high after load with raised ventricular vascular impedance (Zva). Other causes include mitral stenosis and regurgitation, tricuspid regurgitation and atrial fibrillation.

Approximately, one-third of these patients will have pseudo-severe (moderate) aortic stenosis. [34]. DSE (applying the same principles as for classical LF-LG aortic stenosis) may have some role in these patients but the evidence of far less robust. Furthermore the results may be inconclusive, due to the presence of a restrictive myocardial filling. Where DSE is used, the calculation of projected valve area which is less dependent on a large increase in stroke volume may be more robust [34]. Consequently it is critical to integrate other imaging markers of severity of which aortic valve calcium scoring by multi-detector computed tomography has been shown to correlate well with the haemodynamic severity of aortic stenosis $[35,36]$.

Studies have shown that the outcomes of patients with paradoxical LFLG aortic stenosis are better compared with classical LFLG aortic stenosis but worse compared 
with high gradient aortic stenosis. However, survival may be markedly improved by aortic valve replacement compared with conservative management [37,38]. Symptomatic patients with paradoxical low-flow, low-gradient aortic stenosis and evidence of severe aortic stenosis have a class Ila, level of evidence $\mathrm{C}$, indication for aortic valve replacement (ESC/AHA) [3,4].

\section{Performing The Test}

The low dose dobutamine protocol starts at $5 \mathrm{mcg} / \mathrm{kg} / \mathrm{min}$ and increasing in $5 \mathrm{mcg} / \mathrm{kg} / \mathrm{min}$ increments every 5 minutes up to a maximum of $20 \mathrm{mcg} / \mathrm{kg} / \mathrm{min}$. There should be less than 10 beat per minute increase in heart rate. A 12 lead electrocardiogram should be placed to look for ST segment changes and arrhythmia. Blood pressure measurements should be made at each stage. The baseline dataset comprises aortic valve continuous wave Doppler, left ventricular outflow tract pulsed wave Doppler, and views of the left ventricular cavity in apical 4 and 2 chamber positions. These parameters should be repeated at each stage. Changes in LVEF, flow reserve (stroke volume), peak aortic velocity, mean aortic valve pressure gradient and aortic valve area should be calculated [20].

\section{AORTIC REGURGITATION}

The afterload caused by chronic severe aortic regurgitation leads to an elevated left ventricular diastolic pressure and eventual left ventricular dysfunction. Symptomatic patients have an increased risk of sudden death. Exercise testing is recommended to reveal symptoms and aortic valve replacement is considered a Class I (level of evidence 
B) in patients with severe aortic regurgitation and symptoms revealed by exercise testing $[3,4]$.

Exercise stress echocardiography has no role in the assessment of aortic regurgitation severity but can provide an assessment of contractile reserve as well an as objective measure of exercise performance in patients with equivocal symptoms. The evidence for the indication of stress echo for the assessment of contractile reserve is limited. The lack of contractile reserve ( $<5 \%$ change in LVEF) was found to predict left ventricular systolic dysfunction development at follow-up in medically managed patients and post-operatively in those undergoing aortic valve replacement [39]. Failure to increase LVEF at exercise has been found to be specific for predicting left ventricular dysfunction, symptom development or sudden death during follow-up [40,41]. Measurement of contractile reserve may therefore assist in determining the optimal timing of surgery in patients with asymptomatic severe aortic regurgitation, and the absence of contractile reserve may identify the presence of subclinical left ventricular dysfunction compared to resting echocardiography. Markers of subclinical left ventricular dysfunction measured by tissue Doppler imaging and global longitudinal strain can also be measured on stress echocardiography $[42,43]$.

\section{Performing The Test}

The preferred method for image acquisition for exercise echocardiography, is with supine or semi-supine bicycle exercise. Resting and exercise image acquisition include 4 and 2 chamber left ventricular views for calculation of LVEF, estimation of PASP and colour flow Doppler to detect mitral regurgitation. The initial workload is 25 Watts and 
increased in 25 Watt increments every 2 minutes. The test is symptom limited and stopped on development of dyspnoea or fatigue.

\section{CHRONIC PRIMARY MITRAL REGURGITATION}

Myxomatous degenerative mitral valve (MV) disease is the most prevalent cause of primary mitral regurgitation (MR) in developed countries $[44,45,46]$. Historical data suggests prevalence estimates of between $5-15 \%$ with associated morbidity and cardiovascular risk including stroke, atrial fibrillation, heart failure and the need for MV surgery $[47,48]$.

Both European Society of Cardiology (ESC) and American Heart Association/American College of Cardiology (AHA/ACC) provides recommendations on the assessment of MV regurgitation severity and indications for intervention [3,4]. ESC guidelines recommend intervention in symptomatic severe primary regurgitation and LVEF $\geq 30 \%$ (Class IB). In asymptomatic severe primary regurgitation intervention is indicated with evidence of left ventricular dysfunction (left ventricular end-systolic diameter (LVESD) $\geq 45 \mathrm{~mm}$ and/or LV EF $\leq 60 \%$ ) (Class I, B); if LV systolic function is preserved (LV EF $\geq 60 \%$, LVESD $\leq 45 \mathrm{~mm}$ ), atrial fibrillation (AF) or pulmonary hypertension (estimated PASP $>50 \mathrm{mmHg}$ at rest) should prompt consideration of intervention (Class Ila, A). In asymptomatic patients without AF or pulmonary hypertension and with preserved LV systolic function (LV EF $\geq 60 \%$, LVESD 40-44mm) intervention may be considered (Class Ila, A), in cases of low surgical risk $(<1 \%)$ and high likelihood of durable repair (>95\%), with the presence of $\geq 1$ of: flail leaflet / severe left atrial dilatation in sinus rhythm (volume indexed $\geq 60 \mathrm{ml} / \mathrm{m}^{2}$ ). 
In patients with asymptomatic, severe MR, watchful waiting has been considered to be a safe strategy, particularly when conducted in a centre with heart valve expertise and careful follow up [49]. There are however multiple factors influencing outcomes in current trigger-based guidelines. Firstly, the necessity for reliable and reproducible echocardiography to enable accurate serial assessment of left ventricularr trigger based parameters (LVESD and LVEF). In addition, patient adherence to follow-up and prompt reporting of symptoms is important to facilitate timely surgical intervention. Early surgical prior to the onset of symptoms is controversial. Although non-randomized registry data superior outcomes for early intervention compared to medical management, these studies cannot exclude selection bias from their methods $[50,51]$.

\section{THE ROLE Of StRESS Echocardiography}

Exercise stress echocardiography plays a key role in the assessment of MV regurgitation. Stress echocardiography is useful where there is a lack of symptomimaging concordance e.g. symptomatic non-severe MR. In addition, it has an important role in evaluating exercise capacity, clarifying symptom status and risk stratification in patients who appear to be asymptomatic with severe MR. Figure 2 is a algorithm for the use of stress echocardiography in patients with primary mitral regurgitation.

\section{SYMPtomatic MiLd oR MOderate Mitral Regurgitation}

The concept of dynamic MR where there are significant changes in MR severity on exercise is well recognized. Stress echocardiography is useful where symptoms on exertion (dyspnoea) are disproportionate to the severity of MR at rest. Tischler et al [52] demonstrated up to a third of patients with mild MR could develop severe MR on exertion. Therefore exercise stress echocardiography is indicated in patients with dyspnoea on 
exertion with no other cause for their symptoms can be identified to exclude dynamic MR.

\section{Asymptomatic SeVere Mitral Regurgitation}

Stress echocardiography with or without cardiopulmonary exercise testing is a useful objective test to assess symptom status and exercise capacity in patients who are apparently asymptomatic. In a study of 884 asymptomatic or minimally symptomatic patients with significant MR who underwent exercise stress echocardiography, Naji et al [53] found $20 \%$ of the cohort only achieved $<85 \%$ age and sex predicted METS [53]. Lower percentage METS and heart rate recovery in addition to atrial fibrillation, LVEF and right ventricular systolic pressure predicted a composite outcome of death, myocardial infarction, stroke and heart failure. In a separate study, Messika-Zeitoun et al [54] showed, in patients with good functional capacity $(>100 \%$ predicted), delayed surgical intervention by 1 year had no adverse effect on primary outcomes (mortality, myocardial infarction, stroke, and progression to heart failure) and those with reduced ( $\leq 100 \%$ predicted) functional capacity were at higher risk regardless of timing of surgery. Both these studies highlight the value of measuring exercise capacity to determine symptom status.

In addition to exercise capacity, identifying markers of left ventricular impairment in patients with normal LVEF is important. Two-dimensional speckle tracking of longitudinal myocardial deformation with assessment of global longitudinal strain can detect subclinical left ventricular systolic impairment and can be used to assess the presence of contractile reserve. Magne et al [55] found the absence of LV contractile (defined as an increase in global longitudinal strain of $2 \%$ or more) during exercise stress echocardiography in patients with preserved left ventricular function was independently 
associated with a two-fold increase in risk of cardiac events. Similar results were found by Lancellotti et al [56]. In this study, an average global longitudinal strain $>-18.5 \%$ and $a<1.9 \%$ change with exercise are predictive of impaired left ventricular systolic function following surgery [56]. Impaired global longitudinal strain is predictive of poor cardiac outcomes and is associated with all cause mortality; regardless of the presence of left ventricular dysfunction, atrial fibrillation, and the type of MV surgery [57].

Changes in the degree of MR have also been shown to predict future symptom onset. In a study of 61 asymptomatic patients with moderate to severe degenerative MR, patients with a marked increase in effective regurgitant orifice area during exercise $\left(>10 \mathrm{~mm}^{2}\right)$ had lower symptom-free survival than those in whom MR decreased or remained unchanged [58]. In addition, this group found the development of exercise induced pulmonary hypertension (PASP $>60 \mathrm{mmHg}$ ) was associated with significantly reduced 2 year survival of $35 \%$ compared to $75 \%$ in those without exercise induced PASP [59].

Echocardiographic parameters of right ventricular (RV) systolic function and contractile reserve also have value as risk markers for event-free survival in asymptomatic significant MR. Exercise-induced RV dysfunction (exercise tricuspid annular plane systolic excursion $<19 \mathrm{~mm}$ ) and exercise-induced pulmonary hypertension (PASP $>54 \mathrm{~mm} \mathrm{Hg}$ ) both convey incremental prognostic value [60]. Post-surgical follow up of asymptomatic patients undergoing early surgical repair also demonstrates that absence of RV contractile reserve during exercise stress echocardiography predicts lower 5-year event-free survival rates [61]. The use of tricuspid annular plane systolic excursion 
rather than longitudinal RV strain is promoted due to simplicity and reproducibility during exercise.

\section{Test PRotocol}

Exercise echocardiography can be performed either on a semi-upright bicycle or using a treadmill. The advantage of bicycle SE is image acquisition can be performed during exercise rather than post-exercise. Baseline images acquired include: left ventricular volumes, LVEF, global longitudinal strain, MR quantification both visual assessment and quantified using the proximal isovelocity surface area method, PASP. For bicycle SE a typical protocol starts with a workload of 25 Watts. The workload is increased in 25 Watt increments every 2 minutes. The test is symptom limited. Acquisition of images for contractile reserve is performed between a heart rate of $90-110 \mathrm{bpm}$ prior to test cessation. For treadmill SE, images for contractile reserve are obtained immediately post exercise.

\section{SECONDARY MITRAL REGURGITATION}

ESC guidance on intervention in severe secondary/function MR is more conservative reflecting the heterogeneous nature of the pathology and typically advanced underlying left ventricular myocardial disease [3]. There is a class II C recommendation for surgery with severe secondary MR in the context LVEF $>30 \%$ and planned coronary artery bypass. If the LVEF is $<30 \%$ then surgery should be considered (class Ila, C) with symptoms, an option for revascularization and evidence of myocardial viability. In the absence of indication for revascularization, surgery may be considered (Ilb, C) if patients remain symptomatic despite optimal medical therapy (+/- cardiac resynchronization 
therapy) and have a low surgical risk. When surgical risk is not low in this situation then a percutaneous edge-to-edge procedure may be considered (Illb, C) with suitable valve morphology. If symptomatic with LVEF is $<30 \%$ then a heart team discussion should occur and a percutaneous approach may be considered (Ilb, C) following careful evaluation for transplant and ventricular assist device.

In patients with secondary (functional) MR, the role of stress echocardiography is evolving. The balance between MV opening and closing forces alters the degree of MR. These can be influenced by heart rate, preload and afterload. Therefore, in patients with symptoms but only mild or moderate MR exercise stress echocardiography may unmask more severe MR on exercise and explain symptoms [62]. This remains the most useful role in secondary MR. Exercise SE can quantify contractile reserve (regardless of the presence of symptoms), the absence of which is an established risk marker for adverse prognosis in non-ischaemic cardiomyopathies [63]. Quantitative changes in MR severity assessed by exercise SE provide incremental risk stratification with increased risk of cardiac death with increases in effective regurgitant orifice by $\geq 13 \mathrm{~mm}^{2}$ [64]. At present the exact role of these prognostic markers in patient management has not been established.

\section{MITRAL STENOSIS}

The major indication for intervention in mitral stenosis is significant (valve area $<1.5 \mathrm{~cm} 2)$ mitral stenosis in the presence of symptoms [3,4]. Exercise testing is useful to clarify whether patients with severe mitral stenosis are truly asymptomatic. Stress echocardiography useful for haemodynamic assessment of valve severity in patients with symptoms which are disproportionate to resting valve severity. 
The mitral valve orifice area and trans-mitral gradient is dynamic. Exercise in patients with mitral stenosis with restricted mitral valve orifice increases heart rate leading to a reduction in diastolic filling time and increase in trans-mitral gradient which in turn causes an exponential increase in left atrial and pulmonary capillary pressure $[65,66]$. In addition, left atrial compliance modulates the haemodynamic effect of mitral stenosis on the pulmonary vasculature and PASP [67]. These physiological changes explain symptom onset on exercise in mitral stenosis quantified as moderate at rest. However, left atrial pressure and symptoms of dyspnoea are determined by more than just the degree of mitral valve stenosis. Exercise intolerance may often be multifactorial particularly in elderly patients. Additional factors include restrictive lung function, chronotropic incompetence, limited stroke volume reserve may also play a part and need to be considered [66].

Grimaldi et al [68] demonstrated the value of exercise stress ehocardiography in patients with moderate mitral stenosis. In symptomatic patients with only moderate mitral stenosis, 35 patients $(76 \%)$ became short of breath. This was associated with an increase in mean trans-mitral gradient to at least $15 \mathrm{mmHg}$ or PASP $>60 \mathrm{mmHg}$. An example of exercise-induced gradient and PASP changes in a breathless patient with moderate mitral stenosis is provided in figure 3 . If the patient is unable to exercise, DSE can be used safely. At peak dose dobutamine, a mean mitral valve gradient of $\geq 18 \mathrm{mmHg}$ identifies a high risk patient group and can unmask haemodynamic significance in up to $40 \%$ of those with presumed moderate stenosis [69].

The AHA guidelines recommend consideration of percutaneous mitral balloon commissurotomy for symptomatic patients with mitral valve area $>1.5 \mathrm{~cm}^{2}$ if there is 
evidence of hemodynamically significant mitral stenosis based on pulmonary artery wedge pressure greater than $25 \mathrm{~mm} \mathrm{Hg}$ or mean mitral valve gradient greater than 15 $\mathrm{mm} \mathrm{Hg}$ during exercise [4]. The ESC guidelines recommend consideration of percutaneous mitral balloon commissurotomy in symptomatic patients with a valve area $>1.5 \mathrm{~cm}^{2}$ if symptoms cannot be explained by another cause and if the anatomy is favourable [3].

\section{Test PRotocol}

Exercise stress echocardiography is performed on a semi-upright bicycle with a starting workload of 25 Watts. The workload is increased in 25 Watt increments every 2 minutes. The test is symptom limited. Images obtained at rest measure mean MV gradient, degree of MR and PASP. Peak images are obtained during exercise when the patient becomes symptomatic.

\section{CONCLUSION}

Stress echocardiography is an important clinical tool to improve our understanding of the significance of valve disease. Stress echocardiography provides data in four major domains; the obstructivity / compliance of the valve, changes to regurgitant volume, ventricular contractile reserve and the effect on the pulmonary vasculature. In addition to these mechanistic insights it provides an objective measure of exercise capacity. These insights provide additional diagnostic and prognostic information to help risk stratify patients and identify the optimal timing of intervention. 


\section{YEAR VIEW}

The current evidence base for stress echocardiography for valve disease is limited due to a relative small sample sizes studied. Although there are multiple studies showing the prognostic value of stress echocardiography in asymptomatic patients with severe valve regurgitation or stenosis, there is a lack of prospectively designed trials examining the effect of intervention based on these cut-off on clinical outcomes. We propose, large, multi-centre, international registries to allow further validation of results and define cutoffs for intervention more rigorously. This would allow testing of this cut-points in prospective trials of intervention based on stress echocardiography data. 


\section{FIGURES}

Figure 1. Diagnostic Algorithm For The Use Of Stress Echocardiography In Patients With Aortic Stenosis.

Figure 2. Diagnostic Algorithm For The Use Of Stress Echocardiography In Patients With Primary Mitral Regurgitation.

Figure 3. 56 year old patient with rheumatic mitral stenosis who notices they are short of breath on exertion. (Panel $A+B$ ). Resting mean transmitral gradient $7 \mathrm{mmHg}$ and pulmonary artery systolic pressure $26 \mathrm{mmHg}$. After 7 minutes exercise the patient becomes short of breath. (Panel $C+D)$. There is an increase in mean transmitral mitral gradient from $7 \mathrm{mmHg}$ to $26 \mathrm{mmHg}$ and an increase in pulmonary artery systolic pressure from $26 \mathrm{mmHg}$ to $82 \mathrm{mmHg}$. 


\section{REFERENCES}

1. lung $B$, Baron $G$, Butchart EG, et al. A prospective study of patients with valvular heart disease in Europe: the Euro Heart Study on Valvular Heart Disease. Eur Heart J 2003; 24:1231-1243.

2. Osnabrugge RL, Mylotte D, Head SJ et al. Aortic stenosis in the elderly: disease prevalence and number of candidates for transcatheter aortic valve replacement: a meta-analysis and modeling study. J Am Coll Cardiol 2013; 62:1002-1012.

3. Baumgartner H, Falk V, Bax JJ, et al. 2017 ESC/EACTS Guidelines for the management of valvular heart disease. European Heart Journal 2017;38: 27392791.

4. Nishimura RA, Otto CM, Bonow RO, et al. 2014 AHA/ACC guideline on the management of patients with valvuar heart disease. J Am Coll Cardiol 2014; 63:e57- e185.

5. Bhattacharyya S, Hayward C, Pepper J, et al. Risk stratification in asymptomatic severe aortic stenosis: a critical appraisal. Eur Heart J 2012;33:2377-2387.

6. Pellikka PA, Sarano ME, Nishimura RA, et al. Outcome of 622 adults with asymptomatic, hemodynamically significant aortic stenosis during prolonged follow-up. Circulation 2005;111:3290-3295. 
7. Taniguchi $\mathrm{T}$, Morimoto $\mathrm{T}$, Shiomi $\mathrm{H}$, et al. Sudden death in Patients with Severe Aortic Stenosis Observations from the CURRENT AS Registry. J Am Heart Assoc 2018 pii: e008397. doi: 10.1161/JAHA.117.008397

8. Amato MC, Moffa PJ, Werner KE, et al. Treatment decision in asymptomatic aortic valve stenosis: role of exercise testing. Heart 2001; 86:381-386.

9. Das $\mathrm{P}$, Rimington $\mathrm{H}$, Chambers $\mathrm{J}$. Exercise testing to risk stratify in aortic stenosis. Eur Heart J 2005;26:1309-1313.

10. Saeed S, Rajani R, Seifert R, et al. Exercise testing in patients with asymptomatic moderate or severe aortic stenosis. Heart 2018 doi: 10.1136/heartjnl-2018-312939 [Epub ahead of print]

11. Masri A, Goodman AL, Barr T, et al. Predictors of Long-Term Outcomes in Asymptomatic Patients with With Severe Aortic Stenosis and Preserved Left Ventricular Systolic Function Undergoing Exercise Echocardiography. Circ Cardiovasc Imaging 2016 pii: e004689 doi: 10.1161/CIRCIMAGING.116.004689

12. Lancellotti $\mathrm{P}$, Lebois $\mathrm{F}$, Simon $\mathrm{M}$, et al. Prognostic importance of quantitative exercise Doppler echocardiography in asymptomatic valvular aortic stenosis. Circulation 2005;112: 1377-1382.

13. Marechaux S, Ennezat PV, Legette, et al. Left ventricular response to exercise in aortic stenosis. Echocardiography 2007;9:955-959.

14. Lancellotti P, Magne J, Donal E, et al. Determinants and Prognostic Significance of Exercise Pulmonary Hypertension in Asymptomatic Severe Aortic Stenosis. Circulation 2012;126:851-859. 
15. Goublaire C, Melissopoulou M, Lobo D, et al. Prognostic Value of Exercise Stress Echocardiography in Asymptomatic Patients With Aortic Valve Stenosis. JACC Cardiovasc Imaging 2018;11:787-795.

16. Maréchaux S, Ennezat PV, Le Jemtel TH, et al. Left ventricular response to exercise in aortic stenosis: an exercise echocardiographic study: Echocardiography 2007;24:955-959.

17. Van Pelt NC, Stewart RA, Legget ME, et al. Longitudinal left ventricular contractile dysfunction after exercise in aortic stenosis. Heart 2007;93:732-738.

18. Donal E, Thebault C, O'Connor K, et al. Impact of aortic stenosis on myocardial deformation during exercise. Eur J Echocardiogr 2011; 12: 235-41

19. Huded CP, Masri A, Kusunose K, et al. Outcomes in Asymptomatic Severe Aortic Stenosis With Preserved Ejection Fraction Undergoing Rest and Treadmill Stress Echocardiography. J Am Heart Assoc 2018 pii: e007880 doi: 10.1161/JAHA. 117.007880

20. Bhattacharyya S, Khattar R, Chahal N, et al. Dynamic Assessment of Stenotic Valvular Heart Disease by Stress Echocardiography. Circ Cardiovasc Imaging 2013;6:583-589.

21. Lancellotti P, Pellikka P, Budts W, et al. The Clinical Use of Stress Echocardiography in Non-Ischaemic Heart Disease: Recommendations from the European Association of Cardiovascular Imaging and the American Society of Echocardiography. J Am Soc Echocardiogr 2017;30:101-138. 
22. Michelena HI, Margaryan E, Miller FA, et al. Inconsistent echocardiographic grading of aortic stenosis: is the left ventricular outflow tract important? Heart 2013;99:921-931.

23. Minners J, Allgeier M, Gohlke- Baerwolf C. Inconsistencies of echocardiographic criteria for the grading of aortic valve stenosis. Eur Heart J 2008;29:1043-1048.

24. Caballero L, Saura D, Oliva-Sandoval MJ, et al. Three-dimensional morphology of the left ventricular outflow tract: impact on gradin

25. g aortic stenosis severity. J Am Soc Echocardiogr 2017;30:28-35.

26. deFillippi CR, Willett DL, Brickner ME, et al. Usefulness of dobutamine stress echocardiography in distinguishing severe from non-severe valvular aortic stenosis in patients with depressed left ventricular function and low transvalvular gradient. Am J Cardiol 1995;75:191-194.

27. Monin JL, Quere JP, Monchi M, et al. Low-gradient aortic stenosis: operative risk stratification and predictors for long term outcome: a multicenter study using dobutamine stress haemodynamics. Circulation 2003;108:319-324.

28. Pibarot P, Dumesnil JG. Low flow, low gradient aortic stenosis with normal and depressed left ventricular ejection fraction. J Am Coll Cardiol 2012;60:1845-1853.

29. Clavel MA, Burwash IG, Mundigler G. Validation of conventional and simplified methods to calculate projected valve area at normal flow rate in patients with low flow, low gradient aortic stenosis: the multicenter TOPAS (True or Pseudo Severe Aortic Stenosis) study. J Am Soc Echocardiogr 2010;23:380-386. 
30. Annabi MS, Touboul E, Dahou A, et al. Dobutamine Stress Echocardiography for Management of Low-Flow, Low-Gradient Aortic Stenosis. J Am Coll Cardiol 2018;71:475-485.

31. Levy F, Laurent M, Monin JL, et al. Aortic valve replacement for low-flow/low gradient aortic stenosis; operative risk stratification and long term outcome: a European multicentre study. J Am Coll Cardiol 2008;51:1466-1472.

32. Blais C, Burwash IG, Mundigler G, et al. Projected valve area at normal flow rate improves the assessment of stenosis severity in patients with low flow low gradient aortic stenosis: the multicenter TOPAS (Truly or Pseudo Severe Aortic Stenosis) study. Circulation 2006;113:711-721.

33. Hachicha Z, Dumesnil JG, Bogaty P, et al. Paradoxical low flow, low gradient severe aortic stenosis despite preserved ejection fraction is associated with higher afterload and reduced survival. Circulation 2007;115:2856-2864.

34. Eleid MF, Sorajja P, Michelena HI, et al. Flow-gradient patterns in severe aortic stenosis with preserved ejection fraction: clinical characteristics and predictors of survival. Circulation 2013;128:1781-1789.

35. Clavel MA, Ennezat PV, Maréchaux S, et al. Stress echocardiography to assess stenosis severity and predict outcome in patients with paradoxical low-flow, lowgradient aortic stenosis and preserved LVEF. J Am Coll Cardiol Img 2013;6:175183.

36. Messika-Zeitoun D, Aubry MC, Detaint D, et al. Evaluation and clinical implications of aortic valve calcification measured by electron-beam computed tomography. Circulation 2004;110:356-362. 
37. Cueff C, Serfaty JM, Cimadevilla C, et al. Measurement of aortic valve calcification using multislice computed tomography: correlation with haemodynamic severity of aortic stenosis and clinical implication for patients with low ejection fraction. Heart 2011;97:721-726.

38. Clavel MA, Dumesnil JG, Capoulade R, et al. Outcome of patients with aortic stenosis, small valve area and low-flow, low-gradient despite preserved left ventricular ejection fraction. J Am Coll Cardiol 2012;60:1259-1267.

39. Dayan V, Vignolo G, Magne $\mathrm{J}$, et al. Outcome and impact of aortic valve replacement in patients with preserved LV ejection fraction and low gradient aortic stenosis: a meta-analysis. J Am Coll Cardiol 2015;66:2594-2603.

40. Wahi S, Haluska B, Pasquet A, et al. Exercise echocardiography predicts development of left ventricular dysfunction in medically and surgically treated patients with asymptomatic severe aortic regurgitation. Heart 2000;84:606-614.

41. Siemienczuk D, Greenberg B, Morris C, et al. Chronic aortic insufficiency: factors associated with progression to aortic valve replacement. Ann Intern Med 1989;110:587-592.

42. Borer JS, Hochreiter C, Herrold EM, et al. Prediction of indications for valve replacement among asymptomatic or minimally symptomatic patients with chronic aortic regurgitation and normal left ventricular performance. Circulation 1998;97:525-534.

43. Vinereanu D, lonescu AA, Fraser AG. Assessment of left ventricular long axis contraction can detect early myocardial dysfunction in asymptomatic patients with severe aortic regurgitation. Heart 2001;85:30-36. 
44. Kusunose K, Agarwal S, Marwick TH, et al. Decision making in asymptomatic aortic regurgitation in the era of guidelines: incremental values of resting and exercise cardiac dysfunction. Circ Cardiovasc Imaging 2014;7:352-362.

45. Freed LA, Levy D, Levine RA, et al. Prevalence and clinical outcome of mitralvalve prolapse. N Engl J Med 1999;341:1-7.

46. Levy D, Savage D. Prevalence and clinical features of mitral valve prolapse. Am Heart J 1987;113:1281-1290.

47. Savage DD, Garrison RJ, Devereux RB, et al. Mitral valve prolapse in the general population. Epidemiologic features: the Framingham Study. Am Heart J 1983;106:571-576.

48. Nishimura RA, McGoon MD, Shub C, et al. Echocardiographically documented mitral-valve prolapse: long-term follow-up of 237 patients. N Engl J Med $1985 ; 313: 1305-1309$.

49. Duren DR, Becker AE, Dunning AJ. Long-term follow-up of idiopathic mitral valve prolapse in 300 patients: a prospective study. J Am Coll Cardiol 1988;11:42-47.

50. Rosenhek R, Rader F, Klaar U, et al. Outcome of watchful waiting in asymptomatic severe mitral regurgitation. Circulation 2006;113:2238-2244.

51. Kang DH, Park SJ, Sun BJ, et al. Early surgery versus conventional treatment for asymptomatic severe mitral regurgitation: a propensity analysis. J Am Coll Cardiol 2014;63:2398-2407.

52. Suri RM, Vanoverschelde JL, Grigioni F, et al. Association between early surgical intervention vs watchful waiting and outcomes for mitral regurgitation due to flail mitral valve leaflets. JAMA 2013;310:609-616. 
53. Tischler MD, Battle RW, Saha M, et al. Observations suggesting a high incidence of exercise-induced severe mitral regurgitation in patients with mild rheumatic mitral valve disease at rest. J Am Coll Cardiol 1995;25:128-133.

54. Naji P, Griffin BP, Asfahan F, et al: Predictors of long-term outcomes in patients with significant myxomatous mitral regurgitation undergoing exercise echocardiography. Circulation 2014;129:1310-13019

55. Messika-Zeitoun D, Johnson BD, Nkomo V, et al: Cardiopulmonary exercise testing determination of functional capacity in mitral regurgitation: physiologic and outcome implications. J Am Coll Cardiol 2006;47:2521-2527.

56. Magne J, Mahjoub H, Dulgheru R, Pibarot P, Pierard LA, Lancellotti P. Left ventricular contractile reserve in asymptomatic primary mitral regurgitation. Eur Heart J 2014;35:1608-1616.

57. Lancellotti P, Cosyns B, Zacharakis D, et al: Importance of left ventricular longitudinal function and functional reserve in patients with degenerative mitral regurgitation: assessment by two-dimensional speckle tracking. J Am Soc Echocardiogr 2008;21: 1331-1336.

58. Kim HM, Cho GY, Hwang IC, et al. Myocardial Strain in Prediction of Outcomes After Surgery for Severe Mitral Regurgitation. JACC Cardiovasc Imaging 2018 May 11. pii: S1936-878X(18)30297-3. doi: 10.1016/j.jcmg.2018.03.016

59. Magne J, Lancellotti P, Piérard LA. Exercise-induced changes in degenerative mitral regurgitation. J Am Coll Cardiol 2010;56:300-9.

60. Magne J, Lancellotti P, Piérard LA. Exercise Pulmonary Hypertension in Asymptomatic Degenerative Mitral Regurgitation. Circulation 2010;122:33-41. 
61. Kusunose K, Popović ZB, Motoki H, et al. Prognostic significance of exerciseinduced right ventricular dysfunction in asymptomatic degenerative mitral regurgitation. Circ Cardiovasc Imaging 2013;6:167-176.

62. Vitel E, Galli E, Leclercq C, et al. Right ventricular exercise contractile reserve and outcomes after early surgery for primary mitral regurgitation. Heart 2018;104:855860.

63. Bhattacharyya S, Khattar R, Chahal N, Senior R. Dynamic mitral regurgitation: review of evidence base, assessment and implications for clinical management. Cardiol Rev 2015;23:142-147.

64. Waddingham PH, Bhattacharyya S, Zalen JV, Lloyd G. Contractile reserve as a predictor of prognosis in patients with non-ischaemic systolic heart failure and dilated cardiomyopathy: a systematic review and meta-analysis. Echo Research and Practice 2018;5:1-9.

65. Lancellotti P, Troisfontaines $\mathrm{P}$, Toussaint AC,et al: Prognostic importance of exercise-induced changes in mitral regurgitation in patients with chronic ischemic left ventricular dysfunction. Circulation 2003;108:1713-1717.

66. Leavitt $\mathrm{JI}$, Coats $\mathrm{MH}$, Falk RH. Effects of exercise on transmitral gradient and pulmonary artery pressure in patients with mitral stenosis or a prosthetic mitral valve: a Doppler echocardiographic study. J Am Coll Cardiol 1991;17:1520-6.

67. Laufer-Perl M, Gura Y, Shimiaie J, Sherez J, Pressman GS, Aviram G, Maltais S, Megidish R, Halkin A, Ingbir M, Biner S, Keren G, Topilsky Y. Mechanisms of Effort Intolerance in Patients With Rheumatic Mitral Stenosis: Combined 
Echocardiography and Cardiopulmonary Stress Protocol. JACC Cardiovasc Imaging 2017;10:622-633.

68. Schwammenthal E, Vered Z, Agranat O, Kaplinsky E, Rabinowitz B, Feinberg MS. Impact of atrioventricular compliance on pulmonary artery pressure in mitral stenosis: an exercise echocardiographic study. Circulation 2000;102:2378-2384.

69. Grimaldi A, Olivotto I, Figini F, Pappalardo F, Capritti E, Ammirati E, Maisano F, Benussi S, Fumero A, Castiglioni A, De Bonis M, Vermi A, Colombo A, Zangrillo A, Alfieri O. Dynamic assessment of 'valvular reserve capacity' in patients with rheumatic mitral stenosis. Eur Heart J Cardiovasc Imaging 2012;13:476-482.

70. Reis G, Motta MS, Barbosa MM, Esteves WA, Souza SF, Bocchi EA. Dobutamine stress echocardiography for noninvasive assessment and risk stratification of patients with rheumatic mitral stenosis. J Am Coll Cardiol 2004;43:393-401. 